\title{
Ein Volt für alle Fälle? \\ Strategischer Einsatz von Frames zur Legitimation unterschiedlicher Stromnetzausbau-Szenarien
}

\author{
Adriane Schmidt, Sebastian Thuß, Wolfgang Donsbach
}

\begin{abstract}
Kernaussagen
Der Zentralitätsgrad des Energiesystems - und damit der Umfang neu zu errichtender Hochspannungstrassen - variiert je nach Interessenlage unterschiedlicher Akteure. Mit dem Ziel, sowohl für Befürworter eher zentraler als auch dezentralerer Energieversorgung politische Handlungsempfehlungen abzuleiten, wird der Einfluss von vier Botschaften auf die Akzeptanz des Netzausbaus getestet. Eine repräsentative Befragung in München zeigt, dass Männer eher für zustimmende, Frauen hingegen eher für ablehnende Netzausbau-Botschaften empfänglich sind. Die Erfahrung mit einem Stromausfall moderiert die Wirkung einer Botschaft nur tendenziell: Zustimmende Botschaften erhöhen bei Personen mit Stromausfall-Erfahrung die Akzeptanz des Netzausbaus; ablehnende Botschaften allerdings wirken sich nicht negativ auf dessen Akzeptanz aus.
\end{abstract}

\section{Motivation}

Die geplante Energiewende macht einen tiefgreifenden Umbau der energiewirtschaftlichen Infrastruktur nötig. Dabei darf ein kritischer Punkt an individuell empfundener Versorgungssicherheit und Preiswürdigkeit nicht unterschritten werden, da das Gesamtprojekt sonst seine Legitimität zu verlieren droht. Hierbei stellt sich der Aus- und Umbau der Stromnetze als Schlüsselstelle dar: Neue, von den Verbrauchszentren entfernte Erzeugungsregionen müssen an das Übertragungsnetz angeschlossen werden, während das Niederspannungsnetz vom einseitigen Verteiler zur Sammelschiene für dezentrale Einspeiser aufgewertet werden muss (Leprich et al., 2012, S. 48). An dieser Stelle ist jedoch eine Differenzierung angebracht: Nicht alle Größenordnungen, die in der Öffentlichkeit bzgl. des Übertragungsnetz-Ausbaus thematisiert werden, sind durch die Energiewende zweifelsfrei begründbar. Zudem wird der Bedarf an Netzausbau maßgeblich dadurch bestimmt, in welchem Maße dezentrale Konzepte innerhalb der Umgestaltung des Energiesystems eine Rolle spielen. Durch die relativ schnelle und tiefgreifende technologische Umgestaltung des Energiesystems öffnet sich ein Möglichkeitsfenster, innerhalb dessen die bisher dominierenden zentralen Strukturen der Energieumwandlung zugunsten eines noch zu klärenden Anteils dezentraler Versorgung aufgebrochen werden können.

Je nachdem, wie sich das Mischungsverhältnis aus Zentralität und Dezentralität künftig gestaltet, lassen sich unter- schiedliche Implikationen für den Netzausbau ableiten: Eine vergleichsweise zentral strukturierte Energieversorgung fokussiert ertragreiche Standorte und bedarf daher eines starken Netzausbaus, um verbrauchsstarke Regionen anzubinden. Wird die Stromerzeugung nahe des Verbrauchs maximiert, verringert sich der überregionale Netzausbaubedarf (Agora Energiewende, 2013, S. 25). Die in diesem Zusammenhang denkbare Deutung, eine Trasse sei - etwa aufgrund von überdimensionierter Planung - nicht nötig, ermöglicht bei vom Netzneubau Betroffenen ein spezifisches Argumentationsmuster: Unter Verweis auf dezentrale Optionen können Sie sich zwar für die Energiewende, aber gegen das entsprechende Infrastrukturprojekt positionieren (vgl. Schnelle \& Voigt, 2012, S. 32). Entscheidend für die Akzeptanz des Netzausbaus ist daher, dass die Bürger dessen geplanten Umfang als notwendig erachten. Eine Möglichkeit, die Einstellungen zur Notwendigkeit von Stromtrassen zu beeinflussen, ist die Nutzung strategischer Botschaften. Durch den Aufgriff von zustimmenden oder ablehnenden Argumenten kann der Netzausbau in jeweils verschiedene Kontexte gestellt und damit in einer bestimmten Art und Weise gerahmt werden. Die Botschaften werden folglich auch als Frames (,Rahmungen') bezeichnet.

Der vorliegende Beitrag verfolgt zwei Ziele: Zunächst ist von Interesse, inwieweit sich die Erfahrung mit einem Stromausfall auf die Wahrnehmung eines zentralen und damit ggf. anfälligeren Versorgungssystems auswirkt. Zudem werden Botschaften aus dem energiewirtschaftlichen Kontext abgeleitet, die die Akzeptanz des Netzausbaus beeinflussen könnten und auf ihr Beeinflussungspotenzial hin untersucht. Hierbei stellt sich auch die Frage nach zielgruppenspezifischen Unterschieden und Faktoren, welche die Wirksamkeit von bestimmten Botschaften verstärken können bzw. diese abschwächen. Daraus lassen sich einerseits Kommunikationsempfehlungen für eher zentrale als auch für dezentralere Ausgestaltungen der Energiewende ableiten. Andererseits trägt die Sensibilisierung für die Wirkung entsprechender Botschaften und Strategien zu mehr Transparenz innerhalb des Netzausbau-Diskurses bei.

Neben der Analyse, wie wirksam spezifische Frames die Befürwortung des Stromnetzausbaus beeinflussen können, soll mit der Prädisposition ,persönlich erlebte Beeinträchtigung durch einen Stromausfall‘ eine moderierende Variable berücksichtigt werden, für die aufgrund der hohen Versorgungssicherheit in Deutschland noch wenige empirische

1 Dieser Beitrag ist Teil der Forschung innerhalb des Boysen-TU DresdenGraduiertenkollegs, welches von der Friedrich-und-Elisabeth-BoysenStiftung und der TU Dresden gefördert wird. 
Erkenntnisse vorliegen. Die Annahme, dass Versorgungsengpässe im Zuge der Umstellung des Energiesystems zukünftig häufiger eintreten könnten, rechtfertigt die Fokussierung auf diesen Aspekt. Daraus resultierend führten die Verfasser eine repräsentative Telefonbefragung deutschsprachiger Personen ab 18 Jahren in Privathaushalten Münchens durch, wo am 15.11.2012 in etwa der Hälfte der Stadtteile der Strom ausfiel. Damit lag praktisch ein natürliches Experiment vor, bei dem zwei Gruppen zur Verfügung standen, von denen eine dem Stimulus ,Stromausfall‘ ausgeliefert war, die andere nicht. Sowohl vom Stromausfall Betroffene als auch Nicht-Betroffene aus derselben Region können somit hinsichtlich ihrer Einstellungen und möglicher Persuasionsanfälligkeit durch strategische Botschaften verglichen werden.

\section{Potenzial für dezentrale Strukturen}

Der Konsens, auf den sich die Energiewende stützt, ist zwar breit (BMU, 2012, S. 8), jedoch nicht sonderlich konkret. Dies liegt vor allem daran, dass sich hinter dem Begriff Energiewende ein Spektrum an Ausdeutungen verbirgt, welches verschiedene denkbare Handlungskorridore hinsichtlich ihrer konkreten technischen Umsetzung eröffnet (siehe Abb. 1). Einerseits wird die Energiewende mit dem (beschleunigten) Ausstieg aus der Atomkraft gleichgesetzt, zweitens mit der langfristigen Transformation des Energiesystems im Zeichen der ökologischen Nachhaltigkeit assoziiert. ${ }^{2}$ Zudem lässt sich noch eine dritte Zieldimension in der Frage ausmachen, inwieweit ein energiewirtschaftlicher Pfadwechsel von der traditionell eher zentralen hin zu einer stärker dezentralen Energieversorgung als erstrebenswert beurteilt wird. Dieser wird dadurch ermöglicht, dass die Energiewende innerhalb der generell sehr pfadabhängigen Energiewirtschaft vergleichsweise hohe Freiheitsgrade eröffnet. Da der Begriff der Dezentralität gegenwärtig häufig und mit verschiedenen Bedeutungen verwendet wird, erfolgt zunächst eine kurze Definition. Hierdurch lässt sich gleichzeitig der energiewirtschaftliche Hintergrund skizzieren, vor welchem die Diskussion um die Notwendigkeit des Netzausbaus geführt wird.

Abb. 1: Zieldimensionen der Energiewende

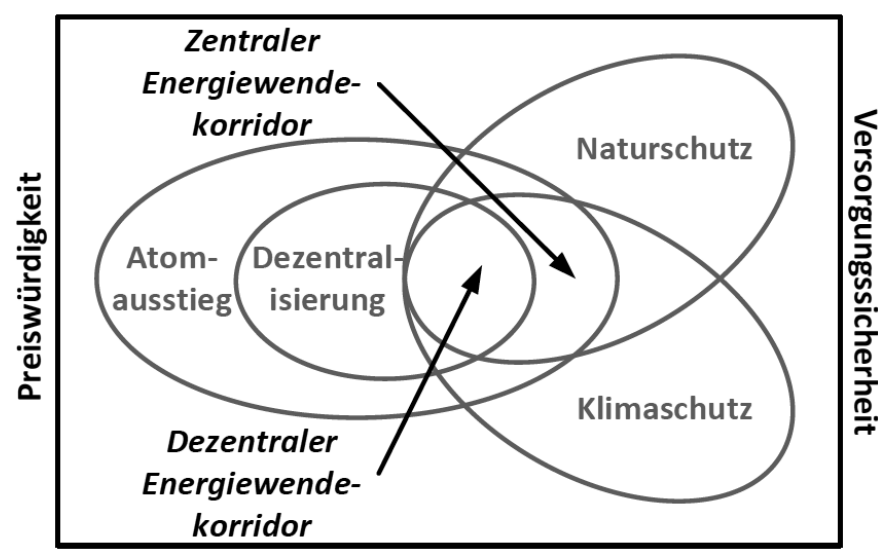

Dezentralität zeichnet sich zunächst durch eine verbrauchsnahe (1) und vergleichsweise ,kleinteilige (2) Energieumwandlung aus, beispielsweise hauseigene Photovoltaik (PV)- und Solarthermieanlagen, ggf. ergänzt durch ebenfalls dezentrale Speicher (Eiselt, 2013, S. 11ff). Zentrale Erneuerbare Energie-Strukturen stellen in dieser Hinsicht vor allem solarthermische Großanlagen dar, wie sie die Desertec Industrial Initiative in Nordafrika plant, sowie die von der Bundesregierung forcierte Offshore-Windkraft (Mautz, 2012, S. 240).

Eine solche Unterscheidung in zentrale und dezentrale Energieversorgung ist jedoch in zweierlei Hinsicht problematisch. Zum einen trägt aus technischer Sicht das deutsche Energiesystem von jeher sowohl zentrale als auch dezentrale Züge. So wurden Kohlekraftwerke nah an Verbrauchszentren errichtet, während Onshore-Windparks im GW-Bereich einen eher zentralen Charakter haben. Wird jedoch das Kriterium der Pluralität der Marktteilnehmer (3) mit in die Definition von Dezentralität einbezogen, so würde auch ein großer Bürgerwindpark als vergleichsweise dezentral gelten. Weiterhin ist Dezentralität nur relativ zu einem überregionalen Bezugssystem (4) zu verstehen. So lässt sich das Muster beobachten, dass jede politische Ebene innerhalb ihres Geltungsgebiets auf Optimierung, das heißt in der Regel Zentralisierung setzt, nach außen aber - je nach eigenem Potenzial - unterschiedlich auftreten kann. Die Bundesrepublik Deutschland verfolgt innerhalb der EU eine vergleichsweise dezentrale Strategie, da sich ihre konkrete Förderpolitik der garantierten Einspeisevergütung von denkbarer EU-weiter Erzeugungsoptimierung (etwa: Solarenergie im MENA-Raum) abgrenzt. Innerhalb Deutschlands forciert die Bundesregierung jedoch einen eher zentralen Technologiepfad, der auf möglichst ertragreiche Standorte (Windkraft v.a. im Norden, PV v.a. im Süden) setzt. Mit Blick auf die lokale Wertschöpfung wiederum möchte die Mehrzahl der Bundesländer die infrage kommenden Technologien derart ausdifferenziert fördern, dass diese künftig auch an wind- und sonnenschwachen Standorten in ganz Deutschland profitabel einsetzbar wären. ${ }^{3}$

Hieraus lässt sich zweierlei folgern: Zunächst ist Dezentralität zwar auch technisch, aber vor allem unter einem Verteilungsaspekt zu betrachten. Dieser wiederum ist direkt mit widerstreitenden Interessen bezüglich des Netzausbaus verbunden, da verschiedene Netzausbauszenarien vor dem Hintergrund des gegenwärtig recht kontingenten Systemumbaus jeweils unterschiedliche energiewirtschaftliche Pfade ,zementieren' würden. Flankiert durch einen zunehmenden Selbstversorgungsanspruch von Bundesländern und Regionen legt

2 Hierbei steht vor allem der - eher globale - Klimaschutz durch verringerte Treibhausgas-Emissionen im Fokus; dies ist weitgehend deckungsgleich mit dem - stärker lokalen - Naturschutz. Angesichts der durchaus vorhandenen ökologischen Belastungen durch die Erneuerbare-Energien-Infrastruktur können hier jedoch durchaus grüngrüne Zielkonflikte auftreten.

3 Vgl. auch Weber \& Hey (2012) zur Richtungsdebatte zwischen harmonisierten Förderinstrumenten auf EU-Ebene und dem Subsidiaritätsprinzip, d.h. angepassten Fördermodellen der Mitgliedsländer. 
der Dreiklang aus Verbrauchsnähe, Kleinteiligkeit und Pluralität an Markteilnehmern ein geringeres Maß an neu zu errichtenden Übertragungsnetzen sowie einen stärkeren Fokus auf zunehmend intelligente Verteilnetze (Nieder-/Mittelspannung) und Speichertechnologien nahe. Einer zu groß dimensionierten Übertragungsnetz-Infrastruktur (Mittel-/Hochspannung) hingegen wird eine Interessenkongruenz mit fossilen Kraftwerken unterstellt (vgl. DIW, 2013; Jarass \& Obermaier, 2012, S. 221). Weiterhin besteht eine offene Flanke im hohen Ausbaubedarf durch den Nord-Süd-Transport von Offshore-Windenergie, welcher zwar regenerativ, hinsichtlich der Besitzstruktur jedoch deutlich als zentral zu bezeichnen ist. Im Umkehrschluss bedeutet dies, dass zu bauenden Hochspannungsnetzen in vielen Fällen zumindest argumentativ eine regenerativ-dezentrale Alternative entgegengestellt werden kann.

Die Argumentationslinie, eine Stromtrasse wäre energiewirtschaftlich nicht notwendig, hat sich im Rahmen von Bürgerprotesten bereits deutlich herauskristallisiert. Als ein Beispiel lässt sich die so genannte Thüringer Strombrücke anführen. Hier stellte die „ungeklärte Notwendigkeit der 380-kvLeitung [...] das größte Hindernis für die Akzeptanz des 380kv-Ausbauprojektes“ dar. Hinterfragt wird dabei nicht die Transformation des Energiesystems an sich, sondern vielmehr, „dass die Leitung nicht ausschließlich diesem Ziel dienen soll bzw. gar nicht notwendig sei, um das Ziel zu erreichen“ (Schnelle \& Voigt, 2012, S. 32). ${ }^{4}$ Kommunikatoren haben somit Spielraum, mit geeigneten Botschaften den Notwendigkeitsdiskurs zu beeinflussen. Dies erfährt besondere Relevanz, wenn der Diskurs von den lokal Betroffenen auf die allgemeine Bevölkerung übergeht.

\section{Framing als Kommunikationskonzept}

Ausgehend vom energiepolitischen Spannungsfeld zwischen zentraler und dezentraler Energieversorgung können Einstellungen zur Notwendigkeit des Netzausbaus durch die Verwendung von Frames beeinflusst werden. Frames entstehen durch die Auswahl und Betonung bestimmter Informationen bei gleichzeitigem Ausschluss anderer Fakten (Entman, 1993; Scheufele, B., 2003). Die Art der Aufbereitung eines Aspekts durch bewusste Informationsselektion kann die Einstellung bzgl. dieses Aspekts in eine bestimmte Richtung beeinflussen und wird als Framing-Effekt bezeichnet (Price, Tewksbury \& Powers, 1997). Eine Vielzahl an Studien, speziell zum Thema Akzeptanz neuer Technologien, konnten bereits FramingEffekte nachweisen (z.B. Cobb, 2005; Shen, 2004; Dardis, 2004; Davis, 1995; Vishwanath, 2009; Stoefs \& Mathijs, 2009).

Bei der Kommunikationsarbeit öffentlicher Akteure spielt das strategische Framing eine zentrale Rolle. Dabei werden Frames als Strategie eingesetzt, um in der entsprechenden Zielgruppe Botschaften zu platzieren und Einstellungen zu manipulieren (Höfelmann, 2013). Gilliam \& Bales (2002) unterscheiden diesbezüglich dominante Frames und Reframes. Während erstere die dominierenden Vorstellungen zu einem
Objekt ausdrücken, stellen Reframes eine bewusste Abkehr und einen Rückgriff auf andere Perspektiven dar, welche durch ihre Neuheit größeres Potenzial haben, die mentalen Vorstellungen zu ändern. „What is needed is a trigger that challenges these mental models and that stimulates consumers to change their minds and develop new mental short cuts" (Stoefs \& Mathijs, 2009, S. 225). Die vorliegende Studie greift diese Konzepte auf und liefert konkrete Handlungsempfehlungen für einen potenziell wirksamen Einsatz beider Typen.

$\mathrm{Ob}$ eine Botschaft eine intendierte Einstellungsbildung oder -änderung hervorruft, hängt von verschiedenen Einflussfaktoren ab. Dabei spielen Prädispositionen eine zentrale Rolle: Sowohl Persönlichkeitseigenschaften, Erfahrungen als auch die soziale Umwelt eines Rezipienten können Einfluss darauf haben, ob und wenn ja, wie stark eine dargebotene Botschaft ihre Wirkung entfaltet. Die vorliegende Studie konzentriert sich neben der individuellen Erfahrung der Betroffenheit auf zwei Persönlichkeitseigenschaften, zu denen bisher wenige Studien vorliegen, die aber entscheidenden Einfluss auf die Wirkung eines Frames haben können (vgl. Matthes, 2007): kognitive Schemata und Zeitpunkt der Urteilsbildung.

\section{Moderator 1: Kognitive Schemata}

Wenn Personen mit neuen Informationen in Berührung kommen, dann wird deren Verarbeitung von bereits vorhandenen Informationen beeinflusst. „An analysis of frames assumes that people are not blank slates when they process new information. They link information they receive to what they already know" (Stoefs \& Mathijs, 2009, S. 224). Diese Informationsbasis hilft dabei, neue Informationen in die vorhandenen „cognitive maps“ (Kaplan \& Kaplan, 1982) einzuordnen (Gilliam \& Bales, 2002). Derartige komplexe Wissenseinheiten bzw. -strukturen werden auch als kognitive Schemata bezeichnet und sind vereinfacht mit den Schubladen eines Schrankes vergleichbar. Es existieren zum einen Rezipienten-Frames bzw. -Schemata, welche die Vorstellungen der Rezipienten ausdrücken, zum anderen die Medien- oder ÖffentlichkeitsFrames, welche in Form einer Kommunikationsbotschaft auf den Rezipienten treffen (Scheufele, 1999).

\section{Moderator 2: Zeitpunkt der Urteilsbildung}

Einstellungen können ,on-line‘ oder erinnerungsgestützt gebildet werden (Bizer et al., 2006). Bei der on-line Urteilsbildung

4 Eine solche Argumentation basiert auf der Auffassung, dass die der Trasse zugrunde liegenden Netzstudien der Deutschen Energieagentur (DENA) die Dekarbonisierung der deutschen Energiewirtschaft sowie die Möglichkeit der Verstärkung bestehender Netze nicht ausreichend berücksichtigen würden (vgl. Schnelle \& Voigt, 2012, S. 14; Jarass \& Obermair, 2012, S. 218 f). Zur Beschleunigung und Legitimitätssteigerung des Netzausbaus wurde zwar im Rahmen des Netzausbaubeschleunigungsgesetzes (NABEG) von 2011 die Erstellung eines Bundesbedarfsplans beschlossen, welcher Bestandteil eines mit vielfachen Beteiligungsschleifen versehenen Netzentwicklungsplans (NEP) sein soll (vgl. BNetzA, 2012, S. 2). Trotz gesteigerter Transparenz und Partizipation finden sich jedoch vor allem seitens von Umweltverbänden nach wie vor die obigen Kritikmuster (vgl. B.U.N.D., 2012, S. 2ff; Deutsche Umwelthilfe, 2011, 3ff; vgl. DIW, 2013). 
werden eingehende Informationen zu einem Objekt sofort verarbeitet und fließen in ein Gesamturteil über das Objekt ein, welches damit während der Informationsverarbeitung gebildet wird. Bei erinnerungsgestützter Urteilsbildung erfolgt lediglich eine Abspeicherung eingehender Informationen. Diese werden genau dann aus der Erinnerung abgerufen und weiterverarbeitet, wenn ein Gesamturteil erforderlich ist, zum Beispiel, wenn eine Person nach ihrem Urteil gefragt wird. Matthes (2007) kritisiert zu Recht, dass die meisten FramingStudien lediglich von einer erinnerungsgestützten Urteilsbildung ausgehen, was einige bereits nachgewiesene FramingEffekte relativiert. Die vorliegende Studie berücksichtigt deshalb gezielt diesen Aspekt.

\section{Moderator 3: Individuelle Erfahrungen: Betroffenheit}

Neben den Persönlichkeitseigenschaften spielt auch die individuelle Erfahrung eine Rolle, wenn es darum geht, wie Frames auf den Rezipienten wirken. Beim Thema Stromnetzausbau bietet es sich an, auf den Aspekt Betroffenheit abzuheben, wobei hier unterschieden werden muss zwischen der Betroffenheit vom konkreten Ausbau (NIMBY-Aspekt) und der Betroffenheit von technischen Störfällen wie der Erfahrung mit einem Stromausfall. Besonders Letzteres ist eng damit verknüpft, wie das Risikopotenzial des Stromnetzes subjektiv eingeschätzt wird. Jungermann und Slovic (1993) argumentieren, dass eine Technik riskanter erscheint, wenn man von einem Schaden im Falle des Versagens direkt betroffen ist oder sein könnte. Dies kann sich zum einen auf die Akzeptanz des Netzausbaus, aber auch konkret auf die Wirkung von strategischen Botschaften auswirken, weshalb dieser Aspekt in der vorliegenden Studie mit besonderem Fokus analysiert wird.

Abb. 2: Untersuchungsmodell mit Moderatoren

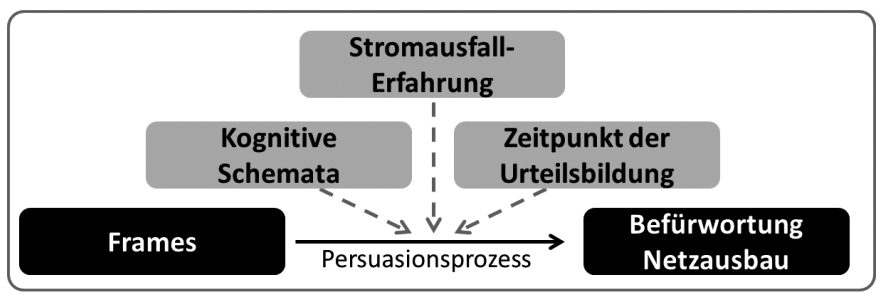

\section{Zielsetzungen und Umsetzung}

\subsection{Forschungsfragen}

Ausgehend von den Potenzialen dezentraler Energieversorgung soll untersucht werden, welchen Einfluss die persönliche Erfahrung mit einem Stromausfall auf die Wahrnehmung einer zentralen Energieversorgung hat: Stehen Beeinträchtigte der zentralen Stromversorgung kritischer gegenüber oder werden zentrale Strukturen eher forciert und Entlastungsmaßnahmen zur Stabilisierung des Netzes befürwortet?

\section{FF1: Inwieweit beeinflusst ein Stromausfall die Wahrnehmung einer zentralen Energieversorgung?}

Im Rahmen der Studie liegt der Schwerpunkt auf der Überzeugungskraft von strategischen Frames sowie der Untersuchung von Faktoren, welche diese Wirkung beeinflussen. Den Einfluss von gezielten Botschaften auf die Akzeptanz eines Einstellungsobjekts konnten bereits mehrere Studien zeigen. Dabei erzielten negativ formulierte Frames stets eine stärkere Wirkung als positive (vgl. Cobb, 2005; Vishwanath, 2009; Davis, 1995). Zudem führte der bewusste Rückgriff auf wenig thematisierte Frames (Reframes) zu stärkeren Framing-Effekten als die Nutzung von dominanten Frames (vgl. Stoefs \& Mathijs, 2009). Hinsichtlich der Formulierung von Frames zeigten die Prinzipien Einfachheit, Verwendung von Metaphern (Rezipienten übernehmen Bildsprache in ihren Formulierungen) sowie Kausalität (Herstellung von Beziehungen und Ursachenzuschreibungen) besondere Effektivität (Van Gorp \& Van der Goot, 2010). Die vorliegende Untersuchung legt den Fokus auf die Tendenz der Frames. Dabei untersuchen die Verfasser, inwieweit sich die Zustimmung zum Netzausbau verändert, wenn der Netzausbau zum einen aus einer positiven Perspektive, zum anderen aus einer negativen Perspektive betrachtet wird. Dies gibt Aufschluss darüber, inwieweit der Frame seine intendierte Wirkung entfaltet.

\section{FF2 a: Welche Überzeugungskraft besitzen die Frames zum Stromnetzausbau?}

Um zielgruppenspezifische Kommunikation zu ermöglichen, fokussieren die Verfasser auf die Untersuchung von drei Moderatoren: (1) Zunächst ist von Interesse, inwieweit die Erfahrung mit einem Stromausfall die Wirkung der Frames auf die Akzeptanz des Netzausbaus beeinflusst. Dabei stellt sich die Frage, ob - besonders im Falle geringerer Akzeptanz des Stromnetzausbaus bei Betroffenen - positive Frames in der Lage sind, die Erfahrung mit negativen Konsequenzen durch einen Stromausfall in gewisser Weise zu kompensieren und (mehr) Akzeptanz für den Stromnetzausbau zu schaffen. Zudem wird überprüft, inwieweit negative Frames bei Betroffenen in der Lage sind, einen geringeren Umfang des Netzausbaus als notwendig zu erachten. Dies liefert zum einen Hinweise, wie Akzeptanz für dezentrale Lösungen geschaffen werden kann; zum anderen Aussagen darüber, wie gefestigt die Meinung zum Stromnetzausbau bei den Bürgern ist. (2) Shen (2004) konnte zeigen, dass die Wirkung von Medien-Frames beim Thema Stammzellenforschung signifikant davon abhängig ist, inwieweit der Frame den kognitiven Schemata eines Rezipienten entspricht. Für die vorliegende Studie soll getestet werden, ob sich diese Ergebnisse auch auf Energiethemen übertragen lassen. Demzufolge müsste bei Personen, deren kognitive Schemata mit einem Frame übereinstimmen, die intendierte Wirkung eines Frames noch stärker ausfallen. Daraus folgt ein vermuteter schwächerer oder gegenläufiger Framing-Effekt, wenn Personen mit einem Frame konfrontiert werden, der nicht den Rezipienten-Schemata entspricht. (3) 
Bizer et al. (2006) konnten eine stärkere Resistenz von on-line gebildeten Urteilen nachweisen. Für die vorliegende Studie soll deshalb untersucht werden, ob im Falle einer erinnerungsgestützten Urteilsbildung positive Frames zu mehr Akzeptanz des Netzausbaus führen, negative Frames zu weniger Akzeptanz und ob eine entsprechende intendierte Wirkung bei online gebildeten Urteilen ausbleibt, was die Resistenz der so gebildeten Einstellungen bestätigen würde. Diese drei Moderatoren werden mit Hilfe der folgenden allgemein formulierten Forschungsfrage untersucht:

\section{FF 2 b: Welche Faktoren verstärken oder verringern die Wirkung der Frames?}

\subsection{Entwicklung der Frames}

Als Input für die Entwicklung der Frames wurde eine qualitative Medieninhaltsanalyse im Zeitraum von Juli bis Dezember 2012 durchgeführt. Mit Hilfe einer Recherche über die Datenbank Factiva wählten die Verfasser Beiträge aus Print- (regional \& überregional) und Online-Medien stichprobenartig aus und durchsuchten diese nach Argumenten für oder gegen den Stromnetzausbau. Einen Schwerpunkt der Analyse bildete die Medienberichterstattung nach dem Stromausfall in München am 15. November 2012 mit dem Ziel, auch negative Argumente bzgl. des Stromnetzausbaus bzw. eines zentralen Stromnetzes aufspüren zu können.

Darauf aufbauend entwickelten die Verfasser zwei PositivFrames, welche befürwortende Argumente des Stromnetzausbaus thematisieren sowie zwei Negativ-Frames, welche durch einen Perspektivwechsel den Stromnetzausbau kritisch beleuchten und einen geringeren Netzumfang einfordern. Die Auswahl der Argumente erfolgte nach der Häufigkeit des Vorkommens, wobei die negative Perspektive in der Berichterstattung eine nur marginale Rolle spielte. Dabei wurden jedoch nur solche Argumente einbezogen, die auf das Spannungsfeld ,Zentralität vs. Dezentralität' Bezug nehmen: ${ }^{5}$ Der EffizienzFrame (+) richtet den Blick darauf, Strom dort zu erzeugen, wo die günstigsten Voraussetzungen herrschen und diesen dann mittels Überlandleitungen an den Verbrauchsort zu transportieren. Der Entlastungs-Frame (+) bezieht sich auf die Beanspruchung des Stromnetzes aufgrund diskontinuierlicher Einspeisung von Sonnen- und Windenergie und einer nötigen Entlastung durch den Netzausbau. Die Positiv-Frames waren medial sehr präsent, sodass sie als dominante Frames bezeichnet werden können. Mit dem Verwundbarkeits-Frame (-) wird das Risiko eines zentralen Stromnetzes aufgezeigt und dezentrale Versorgungsmöglichkeiten als Alternative angeführt. Der Interessen-Frame (-) greift den unterstellten Einfluss konventioneller energiewirtschaftlicher Interessen auf, welcher $\mathrm{zu}$ mehr Netzausbau als nötig führen würde. Befürchtet wird, dass vermehrt Stromleitungen gefordert würden, um die Atom- und Kohlestromproduktion zu ,zementieren' und damit die Macht der großen Stromkonzerne zu sichern. Die beiden Negativ-Frames, insbesondere der Interessen-Frame, haben aufgrund der geringen Thematisierung in den Medien das Potenzial, als Reframes zu fungieren.
Abb. 3: Untersuchte Frames

\begin{tabular}{|c|c|}
\hline $\begin{array}{l}\text { POSITIV: } \\
\text { Effizienz- } \\
\text { Frame }\end{array}$ & $\begin{array}{l}\text { Strom aus Erneuerbaren Energien sollte dort erzeugt werden, wo } \\
\text { viel Sonne scheint oder wo viel Wind weht, z.B. in der Wüste oder } \\
\text { auf dem Meer. Dann muss der Strom aber noch zum Verbraucher } \\
\text { gelangen und dabei zum Teil weite Entfernungen überbrücken. } \\
\text { Wenn man also mehr Stromleitungen bauen würde, könnte der } \\
\text { Strom aus Erneuerbaren Energien besser genutzt werden. }\end{array}$ \\
\hline $\begin{array}{l}\text { POSITIV: } \\
\text { Entlastungs- } \\
\text { Frame }\end{array}$ & $\begin{array}{l}\text { Wenn gerade viel Sonne scheint oder Wind weht, steht viel Strom } \\
\text { zur Verfügung. Dieser kann nicht vollständig in das Stromnetz } \\
\text { eingespeist werden, da es momentan noch nicht für solche Belas- } \\
\text { tungen ausgelegt ist. Wenn man also mehr Stromleitungen } \\
\text { bauen würde, könnte man den Strom aus Erneuerbaren Energien } \\
\text { besser nutzen und das Stromnetz entlasten. }\end{array}$ \\
\hline $\begin{array}{l}\text { NEGATIV: } \\
\text { Verwundbar- } \\
\text { keits-Frame }\end{array}$ & $\begin{array}{l}\text { In Deutschlands großflächigem Stromnetz kann sich ein Defekt } \\
\text { auf größere Teile des Netzes auswirken und zu einem Stromaus- } \\
\text { fall führen. Dieses Risiko ist geringer, wenn Strom direkt dort } \\
\text { erzeugt wird, wo er gebraucht wird, z.B. durch eine Biogasanlage } \\
\text { in der nahen Umgebung. Damit müsste man dann auch weni- } \\
\text { ger Stromleitungen bauen. }\end{array}$ \\
\hline $\begin{array}{l}\text { NEGATIV: } \\
\text { Interessen- } \\
\text { Frame }\end{array}$ & $\begin{array}{l}\text { Die Energiewirtschaft versucht, beim Stromnetzausbau ihre Inte- } \\
\text { ressen durchzusetzen. Sie verlangt nach mehr Ausbau von Strom- } \\
\text { leitungen, um ihre Kohlekraftwerke voll auslasten und den dort } \\
\text { produzierten Strom auch entsprechend transportieren zu kön- } \\
\text { nen. Kohlestrom soll aber zunehmend durch Erneuerbare Ener- } \\
\text { gien ersetzt werden, sodass zukünftig eigentlich weniger Strom- } \\
\text { leitungen gebraucht werden als von der Energiewirtschaft gefor- } \\
\text { dert. }\end{array}$ \\
\hline
\end{tabular}

\subsection{Untersuchungsdesign}

Aufgrund der besonderen Situation des Blackouts in München am 15.11.2012, bei dem nur einige Stadtteile vom Stromausfall betroffen waren, führten die Verfasser dort vom 21.01.-05.02.2013 eine repräsentative Telefonbefragung deutschsprachiger Personen ab 18 Jahren (Stichprobe von $\mathrm{n}=526$ ) in Privathaushalten durch. Damit stehen für nachfolgende Analysen zwei natürlich gegebene Gruppen zur Verfügung, die hinsichtlich ihrer Persuasionsanfälligkeit verglichen werden können: vom Stromausfall Betroffene vs. NichtBetroffene. Um sämtliche (auch unbekannte) Störvariablen zu kontrollieren, welche neben den Frames auch die Akzeptanz des Netzausbaus beeinflussen können (z.B. Geschlecht, sozialer Status, Vorwissen), wurde eine dreifache Randomisierung (TU Dresden, 2009) als Kontrolltechnik angewandt: 1. Zufallsauswahl einer Stichprobe aus der Münchner Bevölkerung, 2. Teilung der Stichprobe per Zufall in 5 etwa gleich große Gruppen und 3. zufällige Zuordnung der Frames zu den Gruppen. Damit lassen sich systematische Unterschiede zwischen den einzelnen Gruppen ausschließen. Durch die vier Frames und eine Kontrollgruppe, welche keinen Frame erhielt, ergaben sich fünf Untersuchungsgruppen mit jeweils etwa 100 Personen. In jeder dieser fünf Gruppen befinden sich durch die Zufallsauswahl sowohl Betroffene als auch Nicht-Betroffene, sodass ein Vergleich zwischen diesen beiden Gruppen für jede Frame-Bedingung möglich ist.

Die vier Gruppen mit Frame-Stimulus wurden anschließend nach ihrer Zustimmung zum vorgelesenen Frame (4-stu-

5 Dies führt dazu, dass sowohl die Aspekte ,Kosten des Netzausbaus sowie die ,Folgen für die Umwelt‘ im Rahmen der negativen Frames nicht berücksichtigt wurden. Frame 4 stellt ein Destillat aus der Literatur dar (vgl. DIW, 2013; Jarass \& Obermaier, 2012, S. 221), weil neben dem Aspekt der Verwundbarkeit des zentralen Netzes kein weiterer negativer Frame in der Berichterstattung auftauchte, welcher sich im Spannungsfeld ,Zentralität vs. Dezentralität' verorten ließe. 
fige Skala) befragt. Diese Frage diente einerseits als Indikator für das vorhandene kognitive Schema des Befragten zum Thema Stromnetzausbau, andererseits hatte sie die Funktion, den Frame weniger auffällig in die Befragung einzustreuen. Dabei wird angenommen, dass eine hohe Zustimmung auf Konsistenz des Frames zu vorhandenen Rezipienten-Schemata hinweist, eine niedrige Zustimmung auf Inkonsistenz. Nach der Zustimmungsfrage folgten zwei kurze und einfache PufferFragen zur Ablenkung vom gesetzten Frame. Abgefragt wurde der Betrag des Stromabschlags und ob ein Ökostromtarif bezogen wird.

Die entscheidende Frage bzgl. der Wirkung der Frames folgte nach den Pufferfragen: „Nun zur letzten Frage über Strom: Zurzeit wird in Deutschland diskutiert, wie viele neue Stromleitungen gebaut werden müssen. Aktuell wird mit 2.800 Kilometern geplant, ${ }^{6}$ das ist ungefähr so weit wie die Luftlinie von München nach Island. Wie viele Stromleitungen sollten Ihrer Meinung nach neu gebaut werden: 2800 km-wie geplant, etwas weniger, deutlich weniger oder gar keine? “ Der Fokus liegt klar auf dem Neubau von Stromnetzen, anstatt deren Umbau. An dieser Stelle kommt der Zeitpunkt der Urteilsbildung ins Spiel. Ein valider Indikator, den Zeitpunkt festzustellen, wann sich die Befragten ein Urteil zum Umfang des Stromnetzes bilden bzw. gebildet haben, ist die benötigte Zeit zur Beantwortung der Frage: Je schneller eine Antwort erfolgt, desto wahrscheinlicher ist, dass ein (on-line) Urteil bereits vorliegt (Hertel \& Bless, 2000; Bizer et al., 2006). Die vorliegende Studie erfasst demzufolge die Dauer des Antwortverhaltens. Dabei wurde gemessen, wie viele Sekunden der Befragte für die Beantwortung der Frage zur Akzeptanz des Netzausbaus benötigt. Aus technischen Gründen begann die Zeitmessung allerdings nicht, nachdem der Interviewer die Frage vorgelesen hatte, sondern bereits davor. Die Vorlesezeit des Interviewers ging demzufolge in die Zeitmessung ein. Dies ist aus Sicht der Autoren aufgrund zweier Betrachtungen unproblematisch: Zum einen dient die Zeit nur als relative Vergleichsgröße; zum anderen kann eine mögliche Verzerrung durch die unterschiedliche Vorlesezeit der Interviewer vernachlässigt werden, da deren Einfluss in allen Teilgruppen als konstant anzunehmen ist. Letztere Annahme liegt einer zufälligen Zuordnung der Frames zu den verschiedenen Versuchsgruppen zugrunde. Damit spiegeln sich beispielsweise längere Vorlesezeiten eines Interviewers in allen Frame-Bedingungen wider; die Verzerrung innerhalb einer Bedingung kann damit ausgeschlossen werden.

Abb. 4: Methodisches Design

\begin{tabular}{|c|c|c|c|c|}
\hline & Indikator für Schema & \multicolumn{2}{|c|}{ Puffer-Fragen } & \\
\hline $\begin{array}{l}\text { Frame B } \\
\text { Frame C } \\
\text { Frame D }\end{array}$ & $\begin{array}{l}\text { Zustimmung: } \\
\text { voll und ganz, } \\
\text { eher, eher nicht, } \\
\text { gar nicht }\end{array}$ & $\begin{array}{l}\text { Abschlag } \\
\text { Strom- } \\
\text { preis pro } \\
\text { Monat }\end{array}$ & $\begin{array}{l}\text { Bezug } \\
\text { Öko- } \\
\text { strom- } \\
\text { Tarif? }\end{array}$ & $\begin{array}{l}\text { Netzausbau } \\
2800 \mathrm{~km} \text {-wie } \\
\text { geplant, } \\
\text { etwas weniger, } \\
\text { deutlich } \\
\text { weniger, } \\
\text { gar keiner }\end{array}$ \\
\hline Kein Frame & & & & \\
\hline
\end{tabular}

\section{Ergebnisse}

Die Befürwortung des Stromnetzausbaus ist in der Stichprobe relativ hoch: So stimmen $46 \%$ der Befragten einem Neubau von Stromleitungen im Ausmaß von $2.800 \mathrm{~km}$ wie geplant zu, etwa $22 \%$ sind für etwas weniger Neubau. Lediglich $16 \%$ plädieren für deutlich weniger bzw. gar keinen Netzausbau. Der relativ hohe Prozentsatz von ,weiß nicht'-Angaben (16\%) könnte ein Indiz für den geringen Wissensstand bzw. die Komplexität des Themas sein. Um zu untersuchen, wie sich die Befürwortung des Netzausbaus zwischen Betroffenen und Nicht-Betroffenen vom Stromausfall unterscheidet, wurde ein Index für den Grad der Betroffenheit gebildet. Dieser drückt die wahrgenommene Beeinträchtigung durch den Stromausfall in verschiedenen individuellen Lebensbereichen aggregiert aus. ${ }^{7}$ Betroffene vom Stromausfall stimmen dem geplanten Umfang des Stromnetzausbaus dabei tendenziell stärker zu als Nicht-Betroffene (U-Test, $\mathrm{p}=0,13$ ). Da $\mathrm{p}>0,05,{ }^{8}$ ist der Unterschied zwischen beiden Gruppen allerdings nicht signifikant.

\section{FF1: Inwieweit beeinflusst ein Stromausfall die Wahrnehmung einer zentralen Energieversorgung?}

Beeinträchtigte stimmen eher dem Effizienz-Frame und dem Entlastungs-Frame zu, welche die zentrale Energieversorgung aus einer positiven Perspektive beleuchten; Nicht-Beeinträchtigte stimmen eher dem Verwundbarkeits- bzw. InteressenFrame zu. Letztere stellen das zentrale Netz kritisch dar und führen dezentrale Strukturen als eine Alternative an. Daraus könnte man schließen, dass der Sicherheitsaspekt bei einem Stromausfall in den Vordergrund rückt und auf den ersten Blick wie ,Verschwörungstheorien' wirkende Anprangerungen der Netzbetreiber wenig fruchtbaren Boden finden. Der Fokus auf Sicherheit bestätigt sich auch darin, dass der Entlastungs-Frame tendenziell umso mehr Zustimmung erfährt, je mehr persönliche Beeinträchtigung durch den Stromausfall gegeben ist $\left(\mathrm{R}_{\text {Spearman }}=0,12, \mathrm{p}=0,25\right)$. Da dieser Frame als ein Indikator für den Erhalt des zentralen Netzes aufgefasst werden kann, könnte man plakativ formulieren, dass die Erfahrung mit einem Stromausfall tendenziell zu einer Befürwortung zentraler Stromversorgung mit entsprechend notwendigem Ausbau des Stromnetzes führt.

6 Zahlen auf Grundlage des NEP 2012. Einige Medienbeiträge griffen diesen Umfang Ende 2012 auf, sodass der Bevölkerung diese Zahl durchaus präsent sein müsste.

7 Dafür gaben die Befragten für zehn Bereiche (z.B. Beleuchtung, Telekommunikationsgeräte, Kühlung oder öffentlicher Nahverkehr) an, ob sie sich in diesen nicht $(=0)$, leicht $(=1)$, mittel $(=2)$ oder stark $(=3)$ beeinträchtigt gefühlt haben. Mittels einer summativen Indexbildung ergab sich für jeden Befragten ein Wert zwischen o (in keinem Bereich beeinträchtigt) und 30 (in allen zehn Bereichen stark beeinträchtigt). Nachfolgende Auswertungen können sich auf zwei Variablen der Betroffenheit beziehen: dichotom ( $0=n i c h t$ beeinträchtigt, >o beeinträch tigt), oder ordinal durch Gruppierung in nicht (0), leicht (1-10), mittel (11-20) und stark (21-30) beeinträchtigt.

8 Für nachfolgende Signifikanztests wird das Signifikanzniveau $\alpha=5 \%$ festgelegt. 
Abb. 5: Frame-Zustimmung: Beeinträchtigte vs. Nicht-Beeinträchtigte

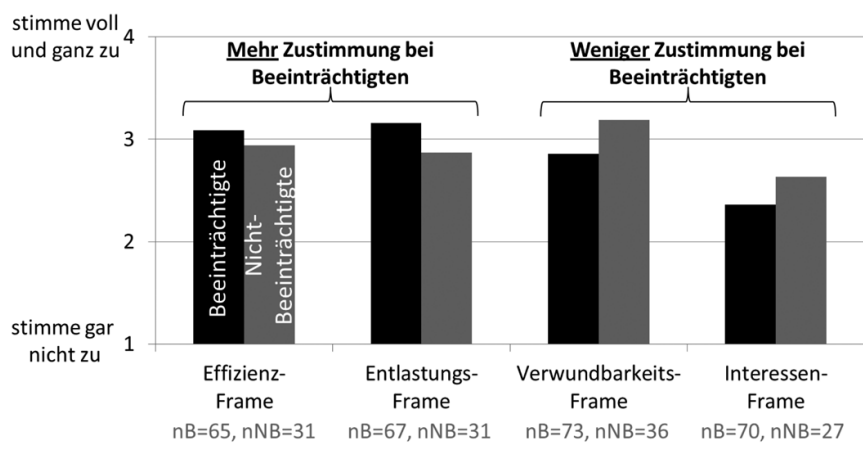

\section{FF2 a: Welche Überzeugungskraft besitzen die Frames zum Stromnetzausbau?}

Befragte, denen positive Frames präsentiert wurden, befürworten den Ausbau des Stromnetzes nicht signifikant stärker als diejenigen, die keinem Frame ausgesetzt waren (U-Test, p=0,30). Beim Effizienz-Frame zeigte sich etwas mehr Akzeptanz des Netzausbaus als beim Entlastungs-Frame, sodass ersterer damit - möglicherweise durch seine Bildhaftigkeit - tendenziell erfolgversprechender ist. Die ausbleibenden signifikanten Framing-Effekte sind ggf. auf die Dominanz der positiven Frames in der öffentlichen Diskussion zurückzuführen, welche damit entweder schon im Vorfeld der Untersuchung eine Wirkung generiert haben oder aber bereits hier schon keinen nennenswerten Einfluss auf die Einstellungen der Bürger zum Netzausbau hatten. Allerdings zeigen sich auch bei den negativen Botschaften keine signifikanten FramingEffekte: Befragte mit negativem Frame-Stimulus zeichnen sich im Vergleich zur Kontrollgruppe nicht durch signifikant geringere Netzausbau-Akzeptanz aus (U-Test, p=0,33). Als effektiver stellte sich der Verwundbarkeits-Frame im Vergleich zum Interessen-Frame heraus. Lediglich Befragte mit positiven und negativen Frame-Bedingungen unterscheiden sich bzgl. der Akzeptanz signifikant (U-Test, $\mathrm{p}=0,02)$.

Abb. 6: Einfluss der Frames auf die Befürwortung des Netzausbaus

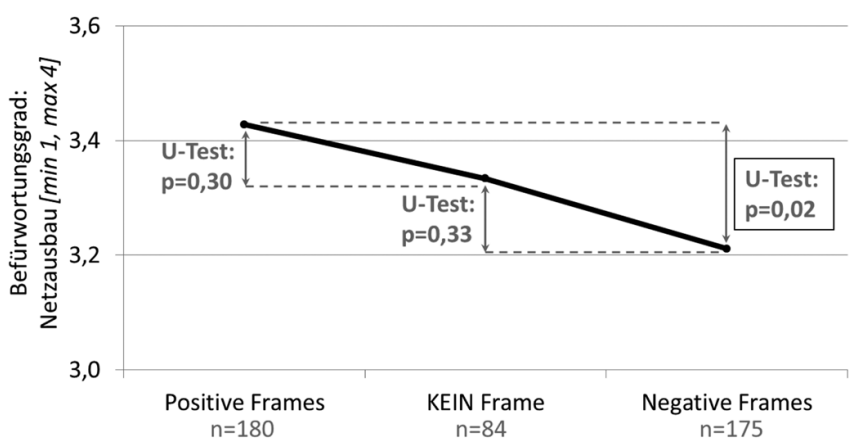

FF 2 b: Welche Faktoren verstärken oder verringern die Wirkung der Frames?

Nachdem sich nur im direkten Vergleich zwischen Befragten mit positiven vs. negativen Frames signifikante Framing-
Effekte gezeigt haben, soll im Folgenden der Fokus auf die drei Prädispositionen in ihrer Moderatorfunktion gelegt werden, deren Berücksichtigung möglicherweise stärkere FramingEffekte zeigt und damit auch die Grundlage für zielgruppenspezifische Ansprachen liefert.

\section{Betroffenheit}

Positive Frames erzeugen bei Betroffenen tendenziell etwas mehr Befürwortung des Netzausbaus im Vergleich zur Kontrollgruppe (U-Test, $\mathrm{p}=0,29)$ als bei Nicht-Betroffenen. Negative Frames verringern die Befürwortung in beiden Gruppen, dies jedoch nicht signifikant. Bei Nicht-Beeinträchtigten haben negative Frames stärkeren Einfluss auf die Akzeptanz des Netzausbaus als bei Beeinträchtigten. Der Interaktionseffekt zwischen Betroffenheit und Frame ist nicht signifikant (ALM Univariat, $\mathrm{F}=0,20, \mathrm{p}=0,82)$. Damit ist das Potenzial relativ gering, dass negative Frames bei Betroffenen eine Meinungsänderung hin zu weniger benötigtem Netzumfang und damit zu weniger Zentralität hervorrufen.

Abb. 7: Moderator ,Stromausfall-Erfahrung auf Framing-Effekt

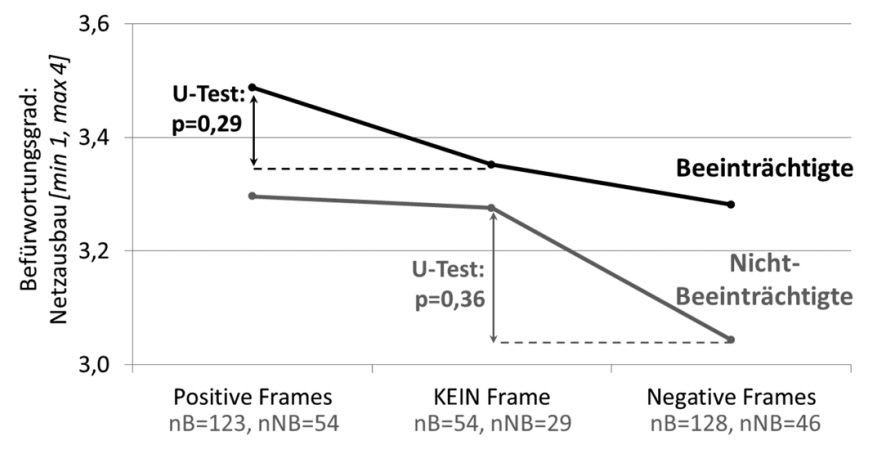

\section{Kognitive Schemata}

Wie bereits festgestellt, befürworten Befragte, denen ein positiver Frame präsentiert wurde, den Ausbau des Stromnetzes nur tendenziell stärker als diejenigen, die keinen Frame erhalten haben. Stimmt allerdings der positive Frame mit dem Rezipienten-Schemata überein, das heißt, wird dem Frame zugestimmt, so zeigt sich ein signifikanter Framing-Effekt (U-Test, $\mathrm{p}=0,02$, (1) ) und die Akzeptanz des Stromnetzausbaus ist deutlich höher als bei Personen ohne Frame-Stimulus. Abb. 8 verdeutlicht, dass die Wirkung des positiven Frames noch einmal verstärkt wird, wenn zwischen dem Frame und dem Rezipienten-Schemata Konsonanz herrscht. Stimmen Befragte einem positiven Frame allerdings nicht zu, zeigt sich ein gegenläufiger Effekt: Befragte zeigen signifikant weniger Akzeptanz für den Netzausbau als Personen, die keinem Frame ausgesetzt waren (U-Test, $\mathrm{p}=0,02$, (2)). Daraus könnte man schließen, dass die untersuchten positiven Frames wenig geeignet sind, mehr Akzeptanz für den Netzausbau zu schaffen, wenn diese nicht den Rezipienten-Schemata entsprechen und damit ein Dissonanz-Verhältnis vorliegt. Analog $\mathrm{zu}$ den positiven Frames zeigen Befragte, die einem negativen Frame ausgesetzt waren, tendenziell eine geringere Akzeptanz des Netzausbaus 
als Befragte ohne Frame. Diese Tendenz wird wieder signifikant, wenn die Rezipienten-Schemata mit dem Frame übereinstimmen und damit Konsonanz vorliegt (U-Test, $\mathrm{p}=0,02$, (3). Wenn einem negativen Frame allerdings nicht zugestimmt wird, dann ist die Akzeptanz des Netzausbaus nicht geringer, sondern geht tendenziell mit mehr Akzeptanz einher (U-Test, $\mathrm{p}=0,16$, (4) - und hat damit keine intendierte Wirkung. Die Ergebnisse machen den wesentlichen Einfluss von kognitiven Schemata auf den Framing-Effekt deutlich. Der Interaktionseffekt von Frame und Rezipienten-Schemata auf die Akzeptanz des Netzausbaus ist hoch signifikant (ALM Univariat, $\mathrm{F}=34,520, \mathrm{p}<0,001)$. Bei den Positiv-Frames ist die Akzeptanz des Netzausbaus umso höher, je mehr die Personen mit dem jeweiligen Frame übereinstimmen $\left(\mathrm{R}_{\text {Spearman }}=0,369 * *\right.$, $\mathrm{p}<0,001)$. Für die Negativ-Frames gilt: Je mehr Zustimmung zum Frame, desto geringer die Akzeptanz des Netzausbaus $\left(\mathrm{R}_{\text {Spearman }}=-0,230^{* *}, \mathrm{p}<0,01\right)$.

\section{Abb. 8: Moderator ,Kognitive Schemata' auf Framing-Effekt}

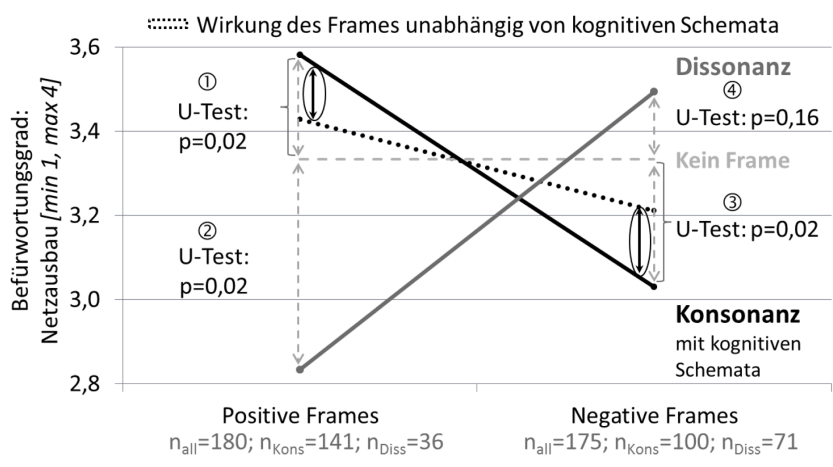

Diese Ergebnisse bedürfen jedoch einer gewissen Relativierung: Als Kontrollgruppe dienen in obigem Fall alle Personen, die keinen Frame erhalten haben. Streng genommen wäre es aber erforderlich, jeweils nur Personen mit den gleichen kognitiven Schemata heranzuziehen und nur bei diesen auf Unterschiede bei der Akzeptanz des Netzausbaus zu testen. Nur so wäre ein eindeutiger Nachweis eines Framing-Effektes möglich. Ob die signifikanten Unterschiede somit auf einen Framing-Effekt zurückzuführen sind oder auf das Vorhandensein der kognitiven Schemata selbst, kann bedingt durch das methodische Design nicht beantwortet werden, da die kognitiven Schemata lediglich bei Personen mit Frame-Stimulus erfasst wurden. Dennoch zeigt sich, dass bei Konsonanz von kognitivem Schemata und gesetztem Frame der Netzausbau stärker (bei positivem Frame) bzw. weniger stark (bei negativem Frame) befürwortet wird als im Vergleich zur Gesamtgruppe ohne Kontrolle der kognitiven Schemata. Dieser anzunehmende verstärkende Effekt von Frames, wenn diese den eigenen Einstellungen entsprechen, konnte bereits bei Shen (2004) gezeigt werden und legt damit die Vermutung nahe, dass es sich bei obigen Ergebnissen tatsächlich um einen Framing-Effekt handeln könnte.

\section{Zeitpunkt der Urteilsbildung}

Die gemessene Zeit zur Beantwortung der Frage nach der Akzeptanz des Netzausbaus in Sekunden wird zu einer dichotomen Variable umcodiert. Dabei dient der Mittelwert (zstandardisiert, um Ausreißer bereinigt) als Trennkriterium. Befragte, welche die Netzausbau-Frage im Schnitt schneller beantworteten, bekommen on-line Urteilsbildung zugewiesen; diejenigen, die länger als der Schnitt benötigten, erinnerungsgestützte Urteilsbildung.

Befragte, die ihre Einschätzung zum benötigten Umfang des Netzausbaus erst bilden, wenn sie durch die vorliegende Untersuchung dazu aufgefordert werden (erinnerungsgestützte Urteilsbildung), zeigen bei den positiven Frames tendenziell mehr ,Anfälligkeit' als Befragte, die bereits im Vorfeld der Untersuchung ein Urteil zum Netzausbau gebildet haben (on-line Urteilsbildung). Im Gegenzug dazu entfalten negative Frames ihre Wirkung eher bei Befragten mit on-line Urteilsbildung, bei denen die Befürwortung des Netzausbaus tendenziell geringer ist als in der Kontrollgruppe (U-Test, $\mathrm{p}=0,09$ ).

Abb. 9: Moderator ,Urteilsbildung' auf Framing-Effekt

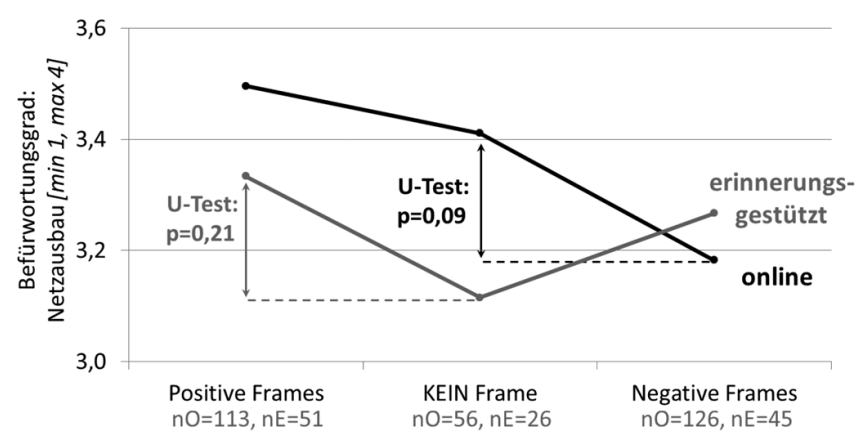

Dass negative Frames bei der on-line Urteilsbildung tendenziell stärkere Framing-Effekte hervorrufen, könnte durch ihre Funktion als Reframes bedingt sein. Durch das bewusste Aufweichen dominanter Strukturen, welche sich in den PositivFrames wiederfinden, ist ein höheres Potenzial für Meinungsbeeinflussung gegeben. Eine weitere Erklärung ist die geringe öffentliche Präsenz der negativen Frames, welche eine Hinterfragung dieser im Vorfeld der Befragung unwahrscheinlich macht. Dies würde das größere ,Störpotenzial' der negativen Frames bis zu einem gewissen Grad erklären. Bei erinnerungsgestützter Urteilsbildung spielt das Reframing vermutlich eine geringere Rolle, da das Urteil erst noch gebildet wird und der präsentierte Frame mit anderen - bereits abgespeicherten Informationen in die Urteilsbildung einfließt. Selbst wenn kognitive Schemata hierbei eine Rolle spielen, haben sich dominante Strukturen bei erinnerungsgestützter Urteilsbildung noch nicht ,festgesetzt'

Auch wenn sich nur Tendenzen zeigen, sollte der Aspekt Zeitpunkt der Urteilsbildung in nachfolgenden Studien verstärkt berücksichtigt werden. Warum beispielsweise Befragte mit erinnerungsgestützter Urteilsbildung bei der Präsentation negativer Frames den Netzausbau stärker befürworten, bleibt zunächst ungeklärt. Möglicherweise beziehen Befragte bei der 
Urteilsbildung die positiven Argumente zum Netzausbau verstärkt ein, die ihnen aufgrund der häufigen Thematisierung (in den Medien) präsenter sind. Dies führt dazu, dass negative Frames dagegen weniger Einfluss und nur bei regelmäßiger Wiederholung und damit Verankerung in den Köpfen der Rezipienten Chance auf Erfolg haben.

\section{Exkurs: Welche Faktoren beeinflussen die Akzeptanz des Netzausbaus?}

Um herauszufinden, welche Faktoren das Antwortverhalten bzgl. des Netzausbaus wie stark beeinflussen, wird eine hierarchische Regression (siehe Abb. 10) durchgeführt. Während der zu Beginn einbezogene Soziodemografie-Block aus Geschlecht, Alter und Bildung etwa 7,2\% der Varianz erklärt, liefert die Integration der Akzeptanz von Erneuerbaren Energien $(\mathrm{EE})^{9}$ nicht signifikant mehr Erklärungskraft. Dies könnte ein Indiz dafür sein, dass ein Zusammenhang zwischen EE und Stromnetz (noch) nicht geknüpft wurde. Damit unterscheiden sich Personen mit Affinität zu EE hinsichtlich der Einschätzung zum benötigten Netzumfang nicht von denen, die EE weniger akzeptieren. Die Erfahrung mit einem Stromausfall hat nur einen geringen Einfluss darauf, inwieweit die Bürger dem Netzumfang zustimmen; auch die Frames haben nur geringe Erklärungskraft und lassen die Modellqualität nur um weitere 1,5\% signifikant ansteigen. Die erklärte Gesamtvarianz von lediglich $10,2 \%(p \leq 0,001)$ zeigt jedoch, dass die Akzeptanz des Netzausbaus von einer Reihe anderer Einflussgrößen abhängig ist, die in zukünftigen Studien verstärkt berücksichtigt werden sollten.

Abb. 10: Hierarchische Regression auf Befürwortungsgrad des Netzausbaus

\begin{tabular}{|l|c|c|c|c|}
\hline \multicolumn{1}{|c|}{ Modell plus Erweiterungsschritt } & $\mathrm{R}^{2}$ & Sig. Änderung $\mathrm{R}^{2}$ & Beta-Koeffizient & Sig. Beta-Koeffizient \\
\hline 1. Soziodemografie & 0,072 & 0,00 & & \\
\hline Geschlecht (0 'weibl.' vs. 1 'männl.') & & & 0,22 & 0,00 \\
\hline Alter & & & $-0,14$ & 0,01 \\
\hline Bildung (0 'geringer' vs. 1 'höher') & & & 0,10 & 0,09 \\
\hline $\begin{array}{c}\text { 2. Akzeptanz-Index: Erneuer- } \\
\text { bare Energien (gering bis hoch) }\end{array}$ & 0,073 & 0,85 & 0,00 & 0,95 \\
\hline $\begin{array}{c}\text { 3. Beeinträchtigung (Stromausfall) } \\
\text { (0 'gar nicht' bis3 'stark') }\end{array}$ & 0,087 & 0,04 & 0,13 & 0,03 \\
\hline $\begin{array}{c}\text { 4. Zeitpunkt der Urteil sbildung } \\
\text { (0 'on-line' vs. 1 'memory-based') }\end{array}$ & 0,087 & 0,66 & 0,02 & 0,76 \\
\hline 5. Frames (0 'positiv' vs. 1 'negativ') & 0,102 & 0,03 & $-0,12$ & 0,03 \\
\hline
\end{tabular}

Eine logistische Regression liefert in Abhängigkeit verschiedener Einflussgrößen die Chance auf Befürwortung des Netzausbaus bzw. einen Faktor, um den die Chance auf Befürwortung steigt, wenn man einer bestimmten Gruppe angehört. Die Befürwortung geht dabei als dichotome ${ }^{10}$ Variable in die Analyse ein. Die Größe Exp (B) zeigt das Chancenverhältnis zur Referenzkategorie (=1), welche jeweils in Klammern angegeben ist (siehe Abb. 11). Am Beispiel Geschlecht haben Männer mit dem Wert $\operatorname{Exp}(B)=2,3$ eine etwa doppelt so hohe Chance, den Netzausbau zu befürworten als Frauen; das Verhältnis ist damit 2,3 (m) zu 1 (w). Dieses Ergebnis ist signifikant, da der untere und obere Wert des 95\% Konfidenzintervalls größer 1 ist. Zudem ist die Chance auf Befürwortung bei vom Strom- ausfall Betroffenen knapp doppelt so hoch wie bei den NichtBetroffenen.

Abb. 11: Logistische Regression auf Befürwortungsgrad des Netzausbaus

\begin{tabular}{|c|c|c|c|}
\hline Einflussgröße & \multirow{2}{*}{$\operatorname{Exp(B)}$} & \multicolumn{2}{|c|}{$95 \%$ Konfidenzintervall für EXP(B) } \\
\cline { 3 - 4 } & & Unterer Wert & Oberer Wert \\
\hline Geschlecht (Ref., weibl.') & 2,268 & 1,290 & 3,985 \\
\hline $\begin{array}{c}\text { Stromausfall-Erfahrung } \\
\text { (Ref., nein') }\end{array}$ & 1,770 & 0,990 & 3,165 \\
\hline Alter & 0,974 & 0,959 & 0,990 \\
\hline Bildung (Ref., geringer') & 0,984 & 0,549 & 1,763 \\
\hline Frames (Ref., positiv') & 0,506 & 0,288 & 0,891 \\
\hline
\end{tabular}

Das Geschlecht ist unter den untersuchten Variablen die bedeutendste Einflussgröße auf die Akzeptanz des Netzausbaus. Dabei befürworten Männer den Netzausbau stärker als Frauen (U-Test, $\mathrm{p} \leq 0,001)$. Ausgehend von den Ergebnissen, dass kognitive Schemata den Framing-Effekt beeinflussen, müssten die untersuchten Frames bei den Geschlechtern unterschiedliche Wirkung haben. Diese Vermutung bestätigt sich: Während Männer mit positivem Frame-Stimulus im Vergleich zur Kontrollgruppe signifikant höhere Akzeptanz des Netzausbaus zeigen (U-Test, $\mathrm{p}=0,03$ ), existiert dieser Effekt bei Frauen nicht. Stattdessen akzeptieren Frauen mit negativem Frame-Stimulus den Netzausbau signifikant geringer als die Kontrollgruppe (U-Test, $\mathrm{p}=0,02$ ), bei Männern wiederum zeigt sich dieser Effekt nicht. Damit wird deutlich, dass sich Framing-Effekte verstärken oder abschwächen, wenn bestimmte Merkmale (z.B. Geschlecht, kognitive Schemata) kontrolliert werden.

\section{Schlussfolgerungen}

\subsection{Konsequenzen für die Kommunikation}

Die vorliegende Studie konnte feststellen, dass etwa $68 \%$ der Befragten den Netzausbau wie geplant oder in etwas geringerem Umfang für gut heißen, sodass die Akzeptanz in der Münchner Bevölkerung relativ hoch ist. Personen, die durch den Stromausfall in München beeinträchtigt waren, befürworten den Netzausbau dabei tendenziell stärker als Personen, die nicht davon betroffen waren. Dies lässt Anzeichen dafür erkennen, dass ein Stromausfall wie dieser eher zu einer Befürwortung zentraler Energieversorgungsstrukturen führt, die mit einem stärkeren Netzausbau einhergehen. Dezentrale Konzepte werden in der Bevölkerung vermutlich noch nicht als sichere Alternativen wahrgenommen, sodass hier noch Kommunikationsbedarf seitens entsprechender Befürworter besteht.

Die vier untersuchten strategischen Frames - Effizienz- (+), Entlastungs- (+), Verwundbarkeits- (-) und Interessen-Frame $(-)$ - zeigten im Bezug zur Kontrollgruppe ohne Frame nur

9 Summativer Index aus fünf einzelnen Items als Indikatoren für die Akzeptanz (siehe Schubert et al., 2013, S. 45-46).

10 Zusammenfassung von ,2.800km-wie geplant" und „etwas weniger" zu Befürwortung sowie "deutlich weniger" und "gar keine“ zu Ablehnung. 
tendenzielle Wirkung: So führten positive Frames zu mehr Akzeptanz des Netzausbaus, negative Frames zu weniger Akzeptanz. Von den drei analysierten Prädispositionen hatten nur die kognitiven Rezipienten-Schemata einen signifikanten moderierenden Einfluss, der aufgrund der vorliegenden Methodik leichter Relativierung bedarf: Die Wirkung einer Kommunikationsbotschaft zum Thema Netzausbau hängt vermutlich davon $a b$, inwieweit der präsentierte Frame den Rezipienten-Schemata entspricht. Liegt Konsonanz vor, so haben positive wie negative Frames noch einmal einen verstärkenden Effekt auf die Befürwortung oder Ablehnung des Netzausbaus. Bei den Moderatoren ,Betroffenheit' und ,Zeitpunkt der Urteilsbildung' ließen sich in dieser Studie nur Tendenzen aufzeigen. Dabei wirken positive Frames stärker bei vom Stromausfall Betroffenen; negative Frames hingegen zeigen bei Betroffenen tendenziell einen geringeren Effekt als bei Nicht-Betroffenen.

Das Geschlecht erwies sich bei der Analyse der Einflussfaktoren auf die Akzeptanz des Netzausbaus als bedeutendster Faktor; Männer sind hierbei eher Befürworter als Frauen. Dies erklärt auch, weshalb positive Frames (entsprechend der kognitiven Schemata) bei Männern stärker wirken als bei Frauen; Frauen sich dagegen wiederum eher von negativen Frames beeinflussen lassen.

Da der benötigte Netzumfang auf politischer Ebene noch nicht hinreichend geklärt ist, erscheint es den Autoren nicht möglich, eindeutige Handlungsempfehlungen auszusprechen; diese variieren je nach Zentralitätsgrad des zukünftigen Energiesystems. Vielmehr ist das Ziel zum einen die Entwicklung eines kommunikativen Leitfadens, der für verschiedene politische Weichenstellungen angewendet werden kann; zum anderen soll aber auch ein Beitrag zur Sensibilisierung für Kommunikationsstrategien relevanter Akteure geleistet werden.

Die Verfasser unterscheiden bei nachfolgenden Empfehlungen zum einen danach, ob Akzeptanz für stärkere Zentralität (mehr Netzausbau) oder Dezentralität (weniger Netzausbau) geschaffen werden soll; zum anderen nach Kommunikationszeitpunkten. Neben Empfehlungen für die alltägliche, kontinuierliche Kommunikation (Standardkommunikation) werden auch solche für eine konkrete Betroffenheitssituation - im vorliegenden Beispiel im Rahmen eines flächendeckenden Stromausfalls - (Notfallkommunikation) gegeben.

Bei der Umsetzung des geplanten Netzumfangs von $2.800 \mathrm{~km}$ ist die Setzung positiver Frames erfolgversprechend für eine höhere Akzeptanz, dies insbesondere bei Männern sowie bei bereits den Netzausbau befürwortenden Personen. Zudem sollten negative Frames von Kritikern bewusst gekontert werden, im optimalen Fall noch bevor ein negatives Argument in den öffentlichen Fokus gerät. Außerdem wäre eine Orientierung an der bildhaft-nachvollziehbaren Gestaltung des Effizienz-Frames für die Konstruktion zukünftiger Botschaften sinnvoll. Auch im Notfall erweist sich das Setzen positiver Frames als sinnvoll, da die individuell erlebte Betroffenheit von einem Stromausfall die Zustimmung zum Netz- ausbau, also zu einem zentralen Energiesystem, tendenziell erhöht und positive Frames zudem als Verstärker fungieren können. Dies insbesondere dann, wenn der Frame mit dem kognitiven Rezipienten-Schemata übereinstimmt.

Soll der Fokus eher auf dezentralen Energiekonzepten liegen, ist die Verwendung von negativen Botschaften, welche beispielsweise die Verwundbarkeit des zentralen Netzes aufzeigen, nur in der Standardkommunikation empfehlenswert, dies insbesondere bei Frauen. Denn wenn Personen direkt von einem Stromausfall betroffen sind, führen negative Botschaften dieser Art vermutlich nicht dazu, dass der Umfang des Netzausbaus kritisch hinterfragt und dezentrale Alternativen in Betracht gezogen werden. Hier deuten sich a) das Primat der Versorgungssicherheit und b) die Ausdeutung Fernleitungen gleich Sicherheit als verbreitete Deutungsroutine an. Zugespitzt lässt sich formulieren: Die vertrauten Strukturen der Energiewirtschaft widerspiegelnd kann sich die Bevölkerung den Transport der Energie über größere Entfernungen und die dadurch implizierten traditionellen Systemstrukturen eher vergegenwärtigen als unabhängigere regionale Versorgungskonzepte. Soll Akzeptanz für dezentrale Lösungen geschaffen werden, wäre es langfristig sinnvoll, das Potenzial dieses Technologiepfades besser zu kommunizieren und als sichere Alternative darzustellen. Beim gegenwärtigen Kenntnisstand der Verbraucher böte ein weiterer Stromausfall dieser Kategorie keinen ausreichenden Resonanzboden für die Etablierung dezentraler Vorstellungen einer Energiewende. Allerdings ermöglicht die Setzung negativer Netzausbau-Frames im Rahmen der Standardkommunikation Chance auf höhere Akzeptanz dezentraler Strukturen. Dabei kann auf das Potenzial des Reframings zurückgegriffen werden, was durch die Verwendung neuer Informationen die dominanten Argumente des zentralen Stromnetzes abschwächt und den Fokus auf neue Aspekte legt.

Abschließend sei jedoch auf die Chance verwiesen, die präsente, aber öffentlich wenig thematisierte Debatte über die Richtungsentscheidung der Energiewende zwischen Zentralität und Dezentralität offensiver zu führen. Erst so könnte der Gegenargumentation mangelnde Notwendigkeit des Infrastrukturprojekts ihre Schärfe genommen werden.

Abb. 12: Handlungsempfehlungen für die öffentliche Kommunikation

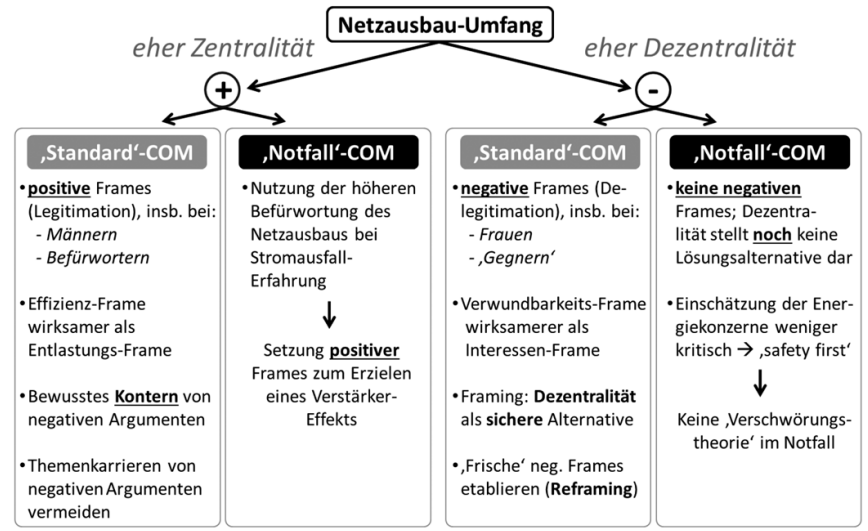




\subsection{Kritische Methoden- \& Ergebnis-Reflexion}

Kritisch anzumerken ist, dass die Befürwortung des Netzausbaus lediglich mit einer Frage operationalisiert wurde. Da Akzeptanz mehrere Dimensionen umfasst, wären mehrere Fragen durchaus valider gewesen. Die Verwendung von lediglich einer Frage war ressourcenbedingt jedoch nicht anders möglich, da diese Untersuchung Teil einer umfassenderen Studie war (siehe Schubert et al., 2013). Zudem ist anzumerken, dass sich der hier vorliegende Untersuchungskomplex im letzten Teil eines umfangreicheren Fragebogens befand, sodass die Auffassungsgabe des Befragten durchaus eingeschränkt gewesen sein könnte. Des Weiteren ist die Art der Pufferfragen zwischen Frame-Stimulus und Abfrage der Akzeptanz als ambivalent zu betrachten. Einerseits sollten sie von dem präsentierten Frame ablenken, andererseits sind eventuell deshalb nur an manchen Stellen signifikante Framing-Effekte nachzuweisen. Zudem ist fraglich, ob die Pufferfrage nach dem Strompreis, welche als leicht zu beantwortende Frage fungieren sollte, nicht bestimmte emotionale Zustände hervorruft, die wiederum einen moderierenden Einfluss auf die FramingEffekte haben könnten.

Viele der hier präsentierten Ergebnisse erwiesen sich als nicht signifikant, was bedingt auch auf die Stichprobengröße von $n=500$ bzw. n=100 pro untersuchter Gruppe zurückzuführen ist. Einige Aspekte wie die Wirkung von FramingEffekten mit Berücksichtigung diverser Einflussfaktoren sollten demzufolge erneut empirisch untersucht werden, um deren Belastbarkeit zu prüfen. Die dargestellten Ergebnisse und daraus abgeleiteten Handlungsempfehlungen für die öffentliche Kommunikation dienen als erste Ansatzpunkte. Für belastbarere Handlungsempfehlungen sind weitere empirische Untersuchungen notwendig. Die vorliegende Studie liefert dennoch erste Eindrücke hinsichtlich der Wirkung von intendierten Botschaften zum Netzausbau und trägt damit zu einem besseren Verständnis von diesbezüglichen Einstellungen bei.

\section{Literaturverzeichnis}

Agora Energiewende (2013): Kostenoptimaler Ausbau der Erneuerbaren Energien in Deutschland. Ein Vergleich möglicher Strategien für den Ausbau von Wind- und Solarenergie in Deutschland bis 2033. Abrufbar unter: http://www.agora-energiewende.de/fileadmin/downloads/presse/Pk_Opti mierungsstudie/Agora_Studie_Kostenoptimaler_Ausbau_der_EE_Web_o ptimiert.pdf (1.12.2013).

Bizer, G. Y., Tormala, Z. L., Rucker, D. D., \& Petty, R. E. (2006). Memory-based versus on-line processing: Implications for attitude strength. Journal of Experimental Social Psychology, 42(5), 646-653.

BMU (2012). Naturbewusstsein 2011. Bevölkerungsumfrage zu Natur und biologischer Vielfalt. Abrufbar unter: http://www.bfn.de/fileadmin/ MDB/documents/themen/gesellschaft/Naturbewusstsein_2011/Naturbe wusstsein-2011_barrierefrei.pdf (1.12.2013).

BNetzA (2012), Bundesnetzagentur legt Entwurf für Bundesbedarfsplan vor. Pressemitteilung vom 26.11.2012. Abrufbar unter: http://www.bun desnetzagentur.de/SharedDocs/Pressemitteilungen/DE/2012/121126_NE PStrom2012Bestaetigung.html (1.12.2013).

B.U.N.D. (2012). Stellungnahme zum Szenariorahmen für den Netzentwicklungsplan 2012. Abrufbar unter: http://www.bund-nrw.de/fileadmi n/bundgruppen/bcmslvnrw/PDF_Dateien/Themen_und_Projekte/Energie _und_Klima/Netzausbau/2011_08_29_BUND_zum_Szenariorahmen_fu er_den_Netzentwicklungsplan_2012.pdf (1.12.2013).
Cobb, M. D. (2005). Framing Effects on Public Opinion about Nanotechnology. Science Communication, 27(2), 221-239.

Dardis, F. E. (2004). Effects of Issue-Framing Functions on Opinion and Intended Behavior: An Experiment Using an Environmental Social Movement Issue. International Communication Association.

Davis, J. (1995). The Effects of Message Framing on Response to Environmental Communications. J\&MC Quarterly, 72(2), 285-299.

Deutsche Umwelthilfe (2011). Stellungnahme zum Entwurf eines Szenariorahmens für den Netzentwicklungsplan 2012. Abrufbar unter: http:// www.duh.de/uploads/media/DUH_Stellungnahme_Netzwentwicklungsp lan.pdf (1.12.2013).

DIW (2013). Netzumbau kein Engpass für die Energiewende. Abrufbar unter http://www.diw.de/de/diw_01.c.421278.de/themen_nachrichten/n etzausbau_kein_engpass_fuer_die_energiewende.html (1.12.2013).

Entman, R. (1993). Framing. Toward clarification of a fractured paradigm. Journal of Communication, 43(4), 51-58.

Eiselt, J. (2013). Dezentrale Energiewende. Wiesbaden: Vierweg + Teubner.

Gilliam, F.D., \& Bales, S.N. (2002). Strategic frame analysis and youth development: how communications research engages the public. In: Lerner, R.M., Jacobs, F. and Wertlieb, D. (Hrsg.): Handbook of Applied Developmental Science: Applying Developmental Science for Youth and Families: Historical and Theoretical Foundations. Thousand Oaks, CA: Sage.

Hertel, G., \& Bless, H. (2000). „On-line“ und erinnerungsgestützte Urteilsbildung: Auslösefaktoren und empirische Unterscheidungsmöglichkeiten. Psychologische Rundschau, 51(1), 19-28.

Höfelmann, M. (2013). Digital Public Affairs: Strategische Kommunikation oder politikferne Selbstvermarktung? In: Roger, F.; Henn, P.; Tuppack, D. (Hrsg.): Medien müssen draußen bleiben! Wo liegen die Grenzen politischer Transparenz? Düsseldorfer Forum Politische Kommunikation. Beiträge zur 8. Fachtagung der DFPK. Berlin: Frank \& Timme.

Jarass, L. \& Obermaier, G. M. (2012). Welchen Netzumbau erfordert die Energiewende? Münster: MV-Verlag.

Jungermann, H. \& Slovic, P. (1993). Charakteristika individueller Risikowahrnehmung. In: Becker, U. (Hrsg.): Risiko ist ein Konstrukt: Wahrnehmungen zur Risikowahrnehmung. München: Knesebeck und Schuler.

Kaplan, S., \& Kaplan, R. (1982). Cognition and Environment: Functioning in an Uncertain World. New York: Praeger Publishers.

Leprich, U. et al.(2012). Kompassstudie Marktdesign. Leitideen für ein Design eines Stromsystems mit hohem Anteil fluktuierender Erneuerbarer Energien. Abrufbar unter: http://www.bee-ev.de/_downloads/publikatio nen/studien/2012/1212_BEE-GPE-IZES-Kompassstudie-Marktdesign.pd f (1.12.2013)

Matthes, J. (2007). Beyond accessibility? Toward an on-line and memorybased model of framing effects. Communications, 32(1), 51-78.

Mautz, R., (2012). Sozio-ökonomische Dynamik der Energiewende. In: Bartelheimer, P.; Fromm, S.; Kädtler, J. (Hrsg.): Berichterstattung zur sozioökonomischen Entwicklung in Deutschland. Teilhabe im Umbruch. Wiesbaden: VS Verlag.

Price, V., Tewksbury, D., \& Powers, E. (1997). Switching Trains of Thought: The Impact of News Frames on Readers' Cognitive Responses. Communication Research, 24(5), 481-506.

Scheufele, B. (2003). Frames-Framing-Framing-Effekte. Wiesbaden: Westdeutscher Verlag.

Scheufele, D. (1999). Framing as a Theory of Media Effects. Journal of Communication, 49, 103-123.

Schnelle, K. \& Voigt, M. (2012). Energiewende und Bürgerbeteiligung. Öffentliche Akzeptanz von Infrastrukturprojekten am Beispiel der „Thüringer Strombrücke“. Abrufbar unter: http://germanwatch.org/de/downl oad/4135.pdf (1.12.2013).

Schubert, D., Meyer, T., von Selasinksy, A., Schmidt, A., Thuß, S., Erdmann, N., Erndt, M. \& Möst, D. (2013): Der Stromausfall in München - Einfluss auf Zahlungsbereitschaften für Versorgungssicherheit und auf die Akzeptanz Erneuerbarer Energien. Schriften des Lehrstuhls für Energiewirtschaft 2. Abrufbar unter http://nbn-resolving.de/urn:nbn:de:bsz:1 4-qucosa-117777 (1.12.2013). 
Shen, F. (2004). Effects of News Frames and Schemas on Individuals' Issue Interpretations and Attitudes. Journalism \& Mass Communication Quarterly, 81(2), 400-416.

Stoefs, E., \& Mathijs, E. (2009). Framing as strategy for effective communication about Fair Trade products in Flanders. International Journal of Agricultural Sustainability, 7(4), 223-234.

TU Dresden (2009). Randomisierung. Abrufbar unter: http://elearning.t u-dresden.de/versuchsplanung/e35/e3824/e3947/ (1.12.2013).

Van Gorp, B., \& Van der Goot, M. (2010). Talking about Sustainability: Responses to Frames in Persuasive Messages about Sustainable Agriculture and Food. International Communication Association.

Vishwanath, A. (2009). From Belief-Importance to Intention: The Impact of Framing on Technology Adoption. Communication Monographs, 76(2), 177-206.

Weber, M. \& Hey, C. (2012). Effektive und effiziente Klimapolitik: Instrumentenmix, EEG und Subsidiarität. Wirtschaftsdienst: Zeitschrift für Wirtschaftspolitik, 92(1), 43-51.

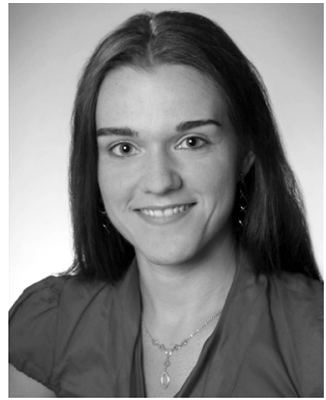

Adriane Schmidt studierte Angewandte Medienforschung an der TU Dresden und ist seit Juli 2012 Promotionsstipendiatin am Boysen-TU Dresden-Graduiertenkolleg. Sie beschäftigt sich dort mit Kommunikationsstrategien für nachhaltige Energiesysteme. Vor Beginn ihrer Promotion war sie Projektmanagerin für Strategische Medieninhaltsanalysen im Bereich Automobil bei PRIME Research in Mainz. E-Mail: adriane.schmidt@tu-dresden.de

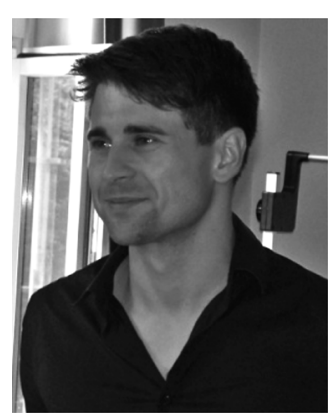

Sebastian Thuß studierte in Politikwissenschaft, Anglistik sowie Neuere und Neueste Geschichte an der TU Dresden und am ITT Dublin. Er ist seit Mai 2012 Promotionsstipendiat am Boysen-TU DresdenGraduiertenkolleg; seine Forschungsschwerpunkte liegen dort auf politischen Steuerungsoptionen und Umsetzungspfaden der Energiewende. E-Mail: sebastian.thuss@tu-dresden.de

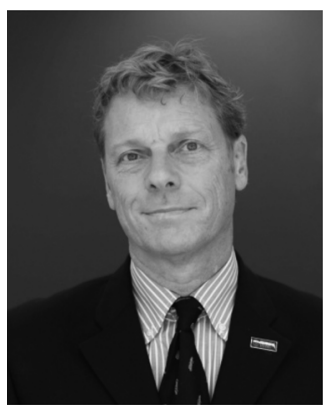

Prof. Dr. Wolfgang Donsbach ist Professor für Kommunikationswissenschaft an der TU Dresden und Gründungsdirektor des dortigen Instituts für Kommunikationswissenschaft; 1995-1996 Präsident der World Association for Public Opinion Research; 2004-5 Präsident der International Communication Association; Fellow der ICA; Forschungsschwerpunkte: Journalismus, öffentliche Meinung, politische Kommunikation. Herausgeber der 12-bändigen International Encyclopedia of Communication. EMail: wolfgang.donsbach@tu-dresden.de 